\title{
Viabilidad del caracol terrestre (Cantareus aspersus) durante la época de descanso
}

\author{
Aragón, M. ${ }^{1} ;$; Angón, E. '; Rodríguez, J. ${ }^{2}$; Barba, C. ${ }^{1}$; Perea, J.. ${ }^{1}$ López, M. ${ }^{3}$ y Cabello, A. ${ }^{1}$ \\ 'Universidad de Córdoba. Campus de Rabanales. Córdoba. España. \\ 2Universidad Técnica Estatal de Quevedo. Ecuador. \\ ${ }^{3}$ Escuela Superior Politécnica Agropecuaria de Manabí. Ecuador.
}

\section{PALABRAS CLAVE ADICIONALES}

Estivación.

Hibernación.

Estrategias productivas.

\section{RESUMEN}

Los periodos de estivación e hibernación del caracol son periodos críticos de en la producción helicícola y su conocimiento contribuye decisivamente a la organización productiva y los resultados económicos de las granjas. El objetivo de este estudio fue determinar la evolución del peso vivo y la viabilidad del caracol terrestre (Cantareus aspersus), en condiciones semicontroladas durante el periodo de reposo. La experiencia tuvo una duración de 32 semanas, desde septiembre de 2013 hasta mayo del 2014. Los resultados obtenidos muestran una disminución del peso vivo del $13,57 \%$ y una perdida de la viabilidad en torno al $20 \%$. Este estudio abre nuevas líneas de investigación orientadas al conocimiento de la fase de hibernación de los caracoles que manejados adecuadamente permite establecer distintas estrategias reproductivas y de manejo en la producción industrial del caracol terrestre C. aspersus.

\section{Viability of land snail (Cantareus aspersus) during resting period}

\section{SUMMARY}

\section{ADDITIONAL KEYWORDS}

Estivation.

Hibernation.

Production strategies.

INFORMACIÓN

Cronología del artículo.

Recibido/received: 11.03 .15

Aceptado/Accept: 13.03.16

Online: 15.09 .16

Correspondencia a los autores/Contact e-mail:

b52armem@uco.es

\section{INTRODUCCIÓN}

La helicicultura en España muestra un auge en los últimos años, de este modo en Andalucía se encuentran registradas más de 50 granjas con una superficie media superior a $2000 \mathrm{~m}^{2}$. Este desarrollo es consecuencia de un crecimiento progresivo del consumo de caracol de crianza y que empieza a considerarse como una alternativa productiva viable en el contexto zootécnico actual y un sistema multiproducto (García et al., 2015). Por otra parte, la carne de caracol puede representar una alternativa en la producción de proteínas para alimentación humana, con bajo impacto ambiental y con revalorización de la dieta mediterránea (Pairotti et al., 2015). De hecho, la carne de caracol es de alto valor nutritivo y de bajo nivel de calorías, con $280 \mathrm{~kJ}$ por cada $100 \mathrm{~g}$ de carne (CREA, 2015).

La estivación e hibernación, según Gallo (2011) y Perea et al. (2006)constituye una fase de gran interés económico y de gran complejidad. En esta fase se determina el éxito de la comercialización y se preparan para su expedición sin que pierdan sus atributos de calidad. El manejo adecuado de la fase de descanso condiciona el éxito productivo de la explotación ya que incide en distintos ámbitos del proceso:

a. Planificación productiva; la hibernación permite hacer lotes de animales y programar las estrategias de reproducción y de descanso dentro de la organización productiva de la explotación de modo que se maximice 
Tabla I. Evolución mensual del peso vivo (Live weight monthly evolution).

\begin{tabular}{|c|c|c|c|c|c|c|c|}
\hline Mes & Promedio & $\begin{array}{c}\text { Desviación } \\
\text { estándar }\end{array}$ & $\begin{array}{c}\text { Coeficiente } \\
\text { de variación }\end{array}$ & Mínimo & Máximo & Rango & $\%$ Viabilidad \\
\hline 1 & $13,36^{c}$ & 3,51 & $26 \%$ & 5,94 & 27,56 & 21,62 & 75 \\
\hline 2 & $12,42^{b}$ & 3,35 & $27 \%$ & 6,73 & 21,63 & 14,90 & 25 \\
\hline 3 & $12,20^{\mathrm{ab}}$ & 3,68 & $30 \%$ & 6,63 & 21,38 & 14,75 & 87,5 \\
\hline 4 & $11,62^{\mathrm{ab}}$ & 3,17 & $27 \%$ & 7,10 & 20,69 & 13,59 & 95 \\
\hline 5 & $10,97^{a}$ & 2,45 & $22 \%$ & 7,66 & 15,27 & 7,61 & 100 \\
\hline 6 & $11,33^{\mathrm{ab}}$ & 2,58 & $23 \%$ & 7,50 & 16,10 & 8,60 & 100 \\
\hline 7 & $11,32^{\mathrm{ab}}$ & 3,09 & $27 \%$ & 4,05 & 16,31 & 12,26 & 91,9 \\
\hline 8 & $12,11^{\mathrm{abc}}$ & 2,10 & $17 \%$ & 9,31 & 15,45 & 6,14 & 87,5 \\
\hline
\end{tabular}

la ocupación del espacio; se incremente la concentración de la puesta/nacimientos y se mejore el tratamiento de animales de acuerdo a su estado productivo.

b. El manejo de la hibernación es clave para establecer buenas prácticas de higiene y profilaxis en la explotación (MARM, 2009).

c. Precios y mercado. La oscilación de precios del caracol es inversa al ciclo productivo de modo que adelantar o posponer la salida de los animales al mercado permite transformar un producto lábil y perecedero y aprovechar un diferencial de precios (Perea, 2007). En este trabajo de carácter preliminar se determina la evolución del peso vivo y la viabilidad de los animales en condiciones naturales semicontroladas durante un periodo de 32 semanas de descanso o estivación.

\section{MATERIAL Y MÉTODOS}

Se utilizaron animales (Cantareus aspersus) procedentes de la Explotación Helicícola Experimental de la Diputación de Córdoba, los cuales permanecieron durante dos semanas en periodo de adaptación con alimentación ad libitum. Posteriormente, se seleccionó una muestra homogénea de 400 caracoles y se identificaron de manera individual. El estudio se llevó a cabo en el laboratorio experimental bajo condiciones semicontroladas, de acuerdo a Bonnet et al. (1990) y García et al. (2006). Se utiliza una hibernación forzada mediante retirada de alimento, evitando la manipulación directa de los animales y el movimiento de sus recintos y además se les proporciona un ambiente seco y aireado aunque sin sol directo. El resto de variables se registran aunque no se modifican los valores naturales (temperatura, humedad, luz, etc.), tan sólo se amortiguan estos valores como consecuencia de la propia nave. La temperatura media durante la experiencia fue de $13,9 \pm 6,9^{\circ} \mathrm{C}$. Los animales se introducen en recipientes cilíndricos de plástico translúcido, a fin de llevar a cabo un seguimiento del crecimiento y la viabilidad durante la hibernación. La experiencia dura 32 semanas; desde el 29 de septiembre de 2013 y hasta el 11 de mayo de 2014. Cada semana, los ejemplares se pesan individualmente dentro su recipiente para evitar su activación. Asimismo semanalmente se separan 10 animales al azar para la evaluación de su viabilidad. Los animales se activan mediante pulverización con agua y se estima la viabilidad productiva como el número de animales que se activan en un periodo de 10 minutos; en tanto que los animales que están muertos o no se activan en dicho periodo son considerados no

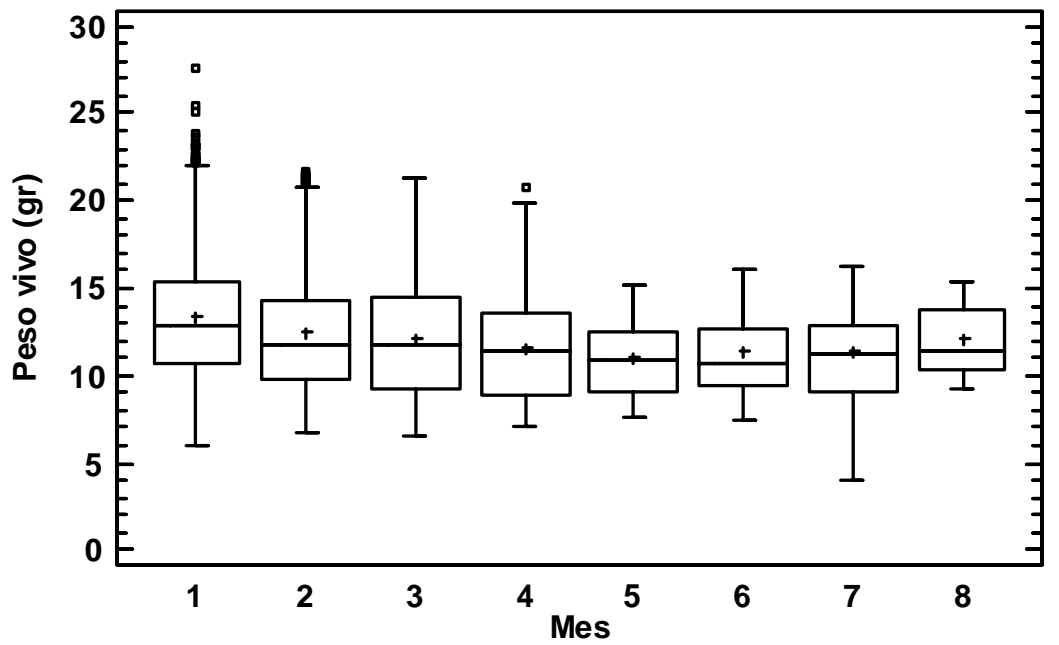

Figura 1. Evolución mensual del peso durante la fase de estivación (Life weight monthly evolution during aestivation). 
viables productivamente. A continuación los diez animales que se activan se retiran de la experiencia.

\section{RESULTADOS Y DISCUSIÓN}

El peso promedio al inicio fue $14,44 \pm 3,69$ gramos y un coeficiente de variación del $25,57 \%$ para finalizar con un peso medio de $11,91 \pm 2,24$ gramos y un coeficiente de variación del 18,82\%, tras los ocho meses de duración de la experiencia. En la tabla I y figura 1 se muestra la evolución mensual de peso. Se observa que el peso disminuye a medida que transcurre el tiempo, aunque de modo contrario a lo esperado, la pérdida de peso fue muy suave, tan sólo de 2,53 gramos en todo el periodo (13,57 \%). La curva muestra dos etapas; una inicial decreciente, durante los dos primeros meses con una disminución brusca del peso $(\mathrm{p}<0,05)$ y posteriormente se mantiene un peso estable durante la experiencia; desde 12,42 $\pm 3,35$ gramos $(27 \%$ CV) hasta 12,11 $\pm 2,10$ gramos $(17 \% \mathrm{CV})$. Asimismo la viabilidad muestra un comportamiento similar; en una primera fase cae bruscamente hasta el $25 \%$ de los animales para luego mantenerse con niveles superiores al $85 \%$. Desde el mes tercero hasta el octavo la situación se mantiene estable; tanto en peso como en viabilidad.

Los resultados muestran que el desarrollo de una hibernación forzada mediante el control de la alimentación, y ventilación como factores clave (el resto de factores estaba dentro de un rango natural aunque amortiguados los extremos) facilita en gran medida el manejo de los animales y evita incurrir en costes operativos e inversiones en la explotación (cámaras frigoríficas, energía, instalaciones y equipos adicionales, etc.). Estos resultados, aunque preliminares, abren nuevas estrategias productivas y reproductivas del caracol en sistemas abiertos y de pronto engorde, tal y como indican Perea (2007) y RAEA (2009).

\section{CONCLUSIONES}

La estivación del caracol terrestre (C. aspersus) durante un periodo de 32 semanas en condiciones semicontroladas reveló resultados favorables, con baja pér- dida de peso y alta viabilidad. La curva de peso y viabilidad muestra dos etapas; una primera decreciente tanto en el peso como la viabilidad, con una duración de dos meses y una segunda etapa sostenida para ambas variables con una duración de seis meses. Los resultados favorables obtenidos en la hibernación forzada en condiciones naturales abren nuevas líneas de estudio y posibles estrategias para la organización productiva y reproductiva de la explotación helicícola.

\section{BIBLIOGRAFÍA}

Bonnet, J.C.; Aupinel, P. et Vrillon, J.L. 1990. L'escargot Helix aspersa, biologie et élevage. INRA. Versailles.

CREA, 2015. Tabelle di composizione degli alimenti. Centro di ricerca per gli alimenti e la nutrizione http://nut.entecra.it/646/ tabelle_di_composizione_degli_alimenti.html?idalimento $=109010 \&$ quant $=100(20 / 02 / 2015)$.

Gallo, G. 2011. El caracol: cría y explotación. Ediciones MundiPrensa. España.

García, A.; Perea, J.M.; Mayoral, A.; Acero, R.; Martos, J.; Gómez, G. and Peña, F. 2006. Laboratory rearing conditions for improved growth of juvenile Helix aspersa Müller snails. Laboratory Animals, 40: 309-316.

García, A.; Murillo, G.; Lara, M. y Rodríguez, D. 2015. Gestión sustentable de empresas agroalimentarias factores clave de estrategia competitiva. Universidad Técnica Estatal de Quevedo. Quevedo.

MARM. 2009. Guía de prácticas correctas de higiene en helicicultura. Ed. Ministerio de Medio Ambiente y Medio Rural y Marino. España. 85 pp.

Pairotti, M.B.; Cerutti, A.K.; Martini, F.; Vesce, E.; Padovan, D. and Beltramo, R. 2015. Energy consumption and GHG emission of the Mediterranean diet: a systemic assessment using a hybrid LCA-IO method. J Clean Prod, 103: 507-516. http://dx.doi.org/ 10.1016/i.jclepro.2013.12.082.

Perea, J. 2007. Factores que afectan al crecimiento del Helix aspersa y sus aplicaciones en helicicultura. Biomodelos de gestión. Tesis Doctoral. Universidad de Córdoba. España.

Perea. J.; Martín, R.; Acero, R.; Félix, E.; Gómez, A.G.; GarcíaMayoral, A.; Peña, F. y García, A. 2006. Selección de hábitat en caracoles terrestres y sus aplicaciones a la helicicultura. Arch Zootec, 55: 1-12.

RAEA. 2009. RAEA Ganadería: Helicicultura. Campaña 2008. Consejería de Agricultura y Pesca. Junta de Andalucía. España. $41 \mathrm{pp}$. 